\title{
Laser Scanning Based Autostereoscopic 3D Display with Pupil Tracking
}

\author{
Erdem Erden, Kishore V. C., Hakan Urey \\ Dept. of Electrical \& Electronics Engineering, Koç University, Istanbul, Turkey \\ eerden@ku.edu.tr \\ Hadi Baghsiahi, Eero Willman, Sally E. Day, David R. Selviah, F. Anibal Fernández \\ Dept. of Electronic \& Electrical Engineering,UCL (University College London), London, U.K \\ Phil Surman \\ De Montfort University, Leicester, U.K
}

\begin{abstract}
An autostereoscopic 3D display based on direct-view RGB laser projection via a transparent display screen is presented. Dynamic exit pupils are formed at the target eye locations with the help of a pupil tracker.
\end{abstract}

Keywords - 3D Display, laser scanning, auto-stereoscopic, dynamic exit pupil, pupil tracking

\section{INTRODUCTION}

Even after years of effort into 3D display technology, a commercially viable 3D display which can offer the true characteristics of a 3D scene is not yet available [1]. This article describes a 3D display which creates $3 \mathrm{D}$ views by projecting images through a low loss transparent display screen while the exit pupil location is dynamically adjusted. This binocular approach eliminates the use of special glasses for viewing the images. The desired images are formed by scanning red, green and blue laser lines on the corresponding liquid crystal on silicon (LCOS) micro-display units. The number of $3 \mathrm{D}$ views is limited by the response time of the micro-display unit.

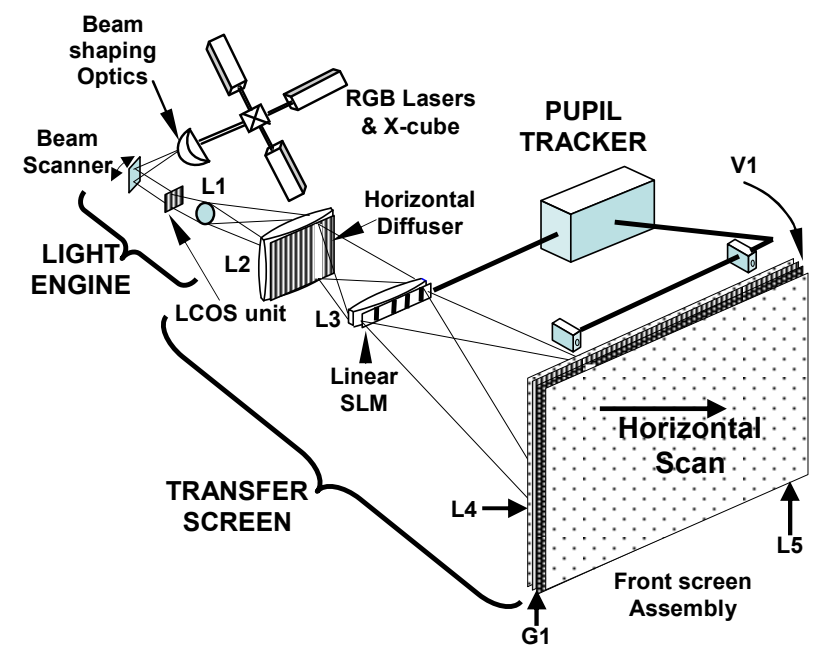

Fig. 1 Simplified schematic drawing of the display system
Fig.1 shows the display optics which are composed of two functional units: the light engine and the transfer screen. The description of the display is given in two sections; the first section discusses the display system in general. The next section includes details of the light engine, modeled by optical design program ZEMAX and some experimental results.

\section{DESCRIPTION OF THE DISPLAY SYSTEM}

Single emitter red (R), green (G), blue (B) lasers with wavelengths $640 \mathrm{~nm}, 532 \mathrm{~nm}$ and $473 \mathrm{~nm}$ respectively are combined with an X-cube and a white laser line is formed. The photopic luminous efficiency functions at the given wavelengths are $0.175,0.885$ and 0.1034 so if the powers of the $\mathrm{R}, \mathrm{G}$, and B lasers are 1: 0.4946: 0.6318 , a white output with a color temperature of $6500 \mathrm{~K}$ can be obtained. The laser line is scanned with a 1D Galvanometer scanner onto the LCOS display units to form columns of images. The LCOS display units can support frame rates up to $100 \mathrm{~Hz}$ and can provide a stereo pair at $50 \mathrm{~Hz}$.

The light column is projected onto the transfer screen of the display system by a multi-element projection lens L1, after modulation. The light column is diffused in the horizontal direction in order for the light from each image column to fill the entire width of the following elements. The aspherical Fresnel lens L2, located before the horizontal diffuser, acts as a field lens to concentrate the axes of the diffused beam towards the centre of the spatial light modulator (SLM) in the horizontal direction. In the vertical direction, it serves to focus the beam on to the SLM. The aspherical Fresnel lens L3 projects the image to the front screen and the SLM controls the direction of the beams exiting the system. A high-speed ferroelectric liquid crystal device is used as the SLM and acts as a dynamic aperture as shown in Fig. 2. The pupil tracking system detects the viewer position in 3D space with the integrated cameras. The transmission of light from the SLM is controlled by the input from the tracker and it is possible to steer the exit pupil according to viewer's eye position in $3 \mathrm{D}$ space. Each image is scanned horizontally across the screen and SLM directs light according to viewer's location dynamically during this process. Left and right images are 
sent to the corresponding eyes by temporal multiplexing so a faster SLM is needed as the number of viewers is increased.
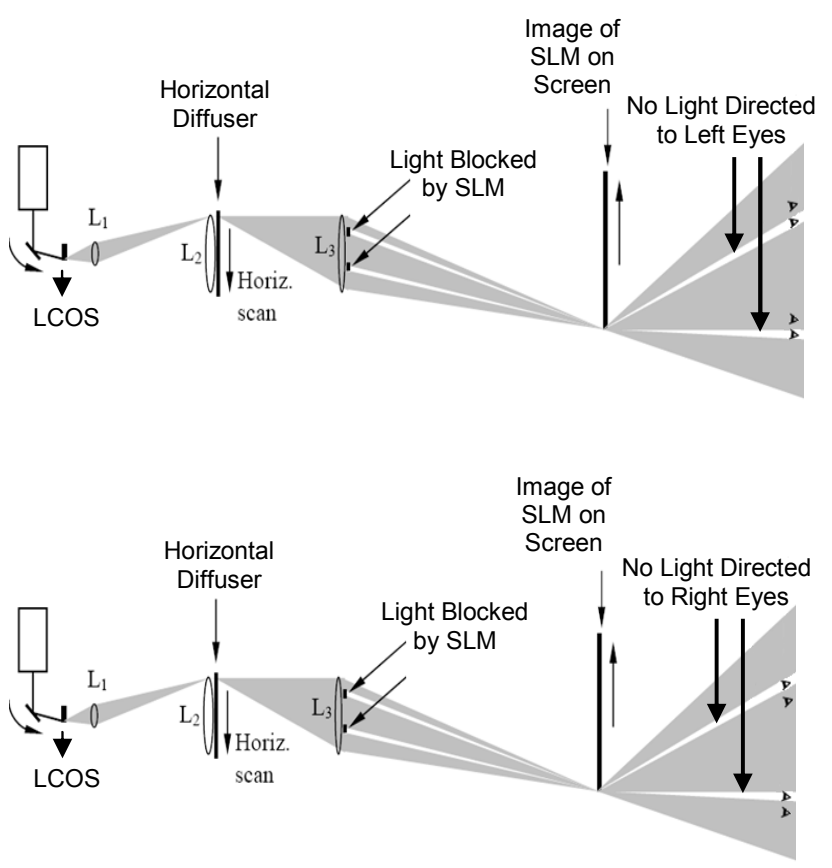

Fig. 2 Side view of the system with dynamic exit pupil control with SLM. SLM blocks the light coming from the diffuser during one full scan for the left eye (upper picture) and for the right eye (lower picture) during the next scan.

After the SLM, light passes through the front screen which consists of aspherical Fresnel lenses L4 and L5, a vertical diffuser V1 and a Gabor superlens assembly [2] G1 with three lenticular microlens arrays. This screen creates an acceptable viewing field by providing an angular magnification to the rays coming from the SLM.

\section{LIGHT ENGINE DESIGN}

We designed systems for both single and multi-emitter lasers [3]. A combined beam from a $4 \mathrm{~W}$ laser (NECSEL) made up of 48 emitters has been characterized experimentally and an optical system for homogenizing and beam shaping designed. One of the sub beams is shown in Fig 3.

A simple prototype of the light engine has been constructed with a $300 \mathrm{~mW}$ single emitter green laser with $514 \mathrm{~nm}$ wavelength. The ZEMAX model of a simplified light engine design from the three lasers to the LCOS unit is shown in Fig. 3. A red laser beam with a $3 \mathrm{~mm}$ beam diameter and $0.8 \mathrm{mrad}$ half divergence angle is combined with green and blue laser with $2 \mathrm{~mm}$ beam diameters and $0.75 \mathrm{mrad}$ half divergence angles with an X-cube. Then the combined beam is expanded with a $5 x$ beam expander and scanned with a $1 \mathrm{D}$ scanner over a $\pm 1.36^{\circ}$ mechanical scan angle. A cylindrical lens with 150 $\mathrm{mm}$ focal length is used to create a line on the LOCS unit. The detector view located at the LCOS plane for three different scan angles is shown in Fig. 3. The colour separation along the scan axis due to different beam sizes is expected to create only a negligible effect as the beam is scanned horizontally.
Perfect overlapping on the LCOS plane can be obtained by using different beam expanders for each laser but this increases both cost and the required volume.

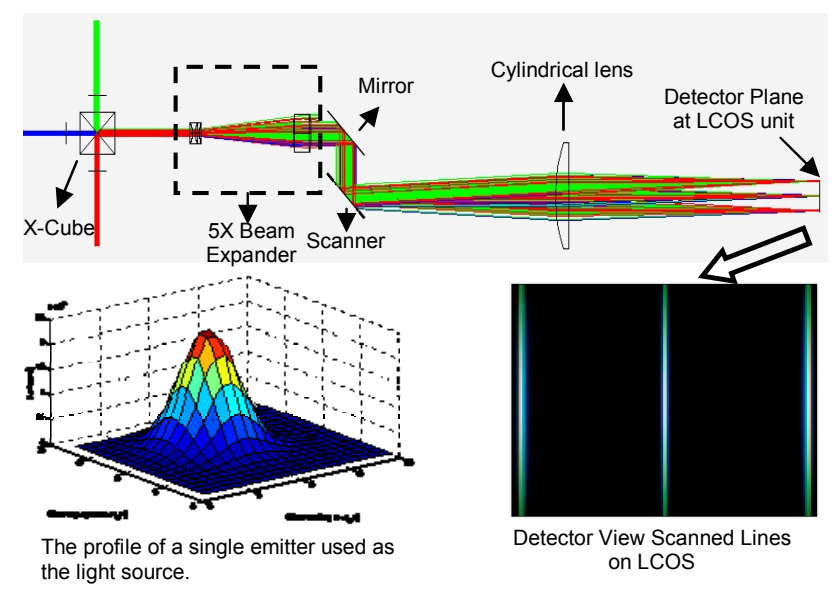

Fig. 3 ZEMAX design of the light engine with three single emitter lasers and the detector output for three different angles

The results of the simple prototype can be seen in Fig.4. The artifacts on the screen are due to the scattered light from the optical components.

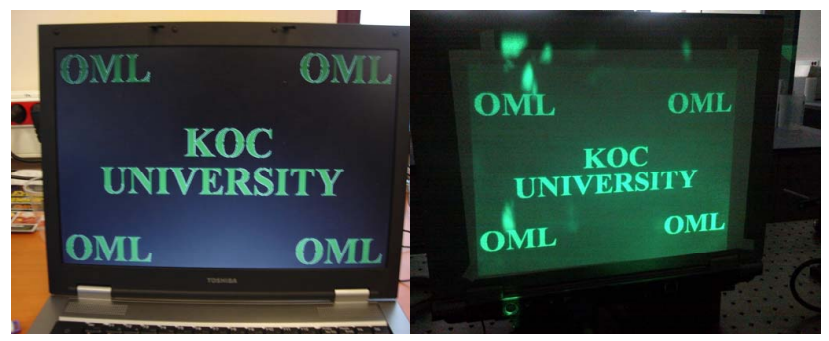

Fig. 4 The original image with green letters on the black computer screen (left) and the laser scanned image on the A4 size white screen (right)

\section{CONCLUSIONS}

An autostereoscopic laser projection based 3D display with pupil tracking is being developed. The system architecture, the design of light engine with single emitter lasers and the results from a single colour demonstration are presented. The first working prototype demonstration of the full system is planned to be finished by the end of 2009 .

\section{ACKNOWLEDGMENT}

Financial support for this project is provided by EC within FP7 under grant 215280 HELIUM3D project. Help from all the members of the HELIUM3D is gratefully acknowledged.

\section{REFERENCES}

[1] P. Benzie, J. Watson, P. Surman, I. Rakkolainen, K. Hopf, H. Urey, V. Sainov, C. von Kopylow, "A Survey of 3DTV Displays: Techniques and Technologies," IEEE Transactions on Circuits and Systems for Video Technology, vol. 17, pp. 1647-1658, 2007.

[2] D. Gabor, UK Patent 541 753, 1940.

[3] K. Chellephan et al, "Laser Scanning 3D Display with Dynamic Exit Pupil," Eurodisplay, Sep 2009 (accepted). 